\title{
Fractional Quantum Hall Effect in a Diluted Magnetic Semiconductor
}

\author{
C. Betthausen ${ }^{1}$, P. Giudici ${ }^{1}$, A. Iankilevitch ${ }^{1}$, C. Preis ${ }^{1}$, V. Kolkovsky ${ }^{2}$, M. Wiater ${ }^{2}$, G. \\ Karczewski ${ }^{2}$, B. A. Piot ${ }^{3}$, J. Kunc ${ }^{3}$, M. Potemski ${ }^{3}$, T. Wojtowicz ${ }^{2}$, and D. Weiss ${ }^{1 \star 1}$ \\ ${ }^{11}$ Department of Experimental and Applied Physics, \\ University of Regensburg, 93040 Regensburg, Germany. \\ ${ }^{2}$ Institute of Physics, Polish Academy of Sciences, 02668 Warsaw, Poland. \\ ${ }^{3}$ Laboratoire National des Champs Magnétiques Intenses, \\ CNRS-UJF-UPS-INSA, 38042 Grenoble, France.
}

(Dated: 21 Mai 2014)

\begin{abstract}
We report the observation of the fractional quantum Hall effect in the lowest Landau level of a two-dimensional electron system (2DES), residing in the diluted magnetic semiconductor $\mathrm{Cd}_{1-x} \mathrm{Mn}_{x}$ Te. The presence of magnetic impurities results in a giant Zeeman splitting leading to an unusual ordering of composite fermion Landau levels. In experiment, this results in an unconventional opening and closing of fractional gaps around filling factor $\nu=3 / 2$ as a function of an in-plane magnetic field, i.e. of the Zeeman energy. By including the $s$ - $d$ exchange energy into the composite Landau level spectrum the opening and closing of the gap at filling factor $5 / 3$ can be modeled quantitatively. The widely tunable spin-splitting in a diluted magnetic 2DES provides a novel means to manipulate fractional states.
\end{abstract}

PACS numbers: 
The fractional quantum Hall effect (FQHE) is a collective high-magnetic field phenomenon, originating from Coulomb repulsion of electrons confined in two dimensions. At certain fractional filling $\nu=p / q$ of the Landau levels (LLs) $[\nu=$ filling factor, $p, q=$ integers], quantized plateaus in the Hall resistance $\rho_{x y}$ and vanishing longitudinal resistance $\rho_{x x}$ herald the presence of peculiar electron correlations $[1,2]$. Here, the electrons condense into a liquid-like ground state that is separated by a gap $\Delta$ from the excited states. Most experiments to date have been carried out on GaAs-based systems, being still the cleanest material system with the highest electron mobilities [3]. When the direction of the magnetic field $B$ is tilted, the orbital LL splitting is given by the field component $B_{\perp}$ normal to the 2DES while the total field strength $B$ determines the Zeeman splitting $E_{Z}$. Early experiments on GaAs revealed that the $\nu=4 / 3,5 / 3$ and $8 / 5$ states behave differently upon tilting the sample $[4,5]$ : While the $\nu=4 / 3$ and $8 / 5$ states were undergoing a transition from a spin-unpolarized state to a polarized one, the $\nu=5 / 3$ state is always fully spin polarized.

Although the FQHE has been reported in quite a number of different materials [6-12], the FQHE was never observed in a diluted magnetic semiconductor in which atoms with magnetic moment (e.g. $\mathrm{Mn}^{2+}$ ) are placed in the 2DES. Then, the localized spins in the magnetic impurities' $d$-orbitals interact with the correlated electron system via the quantum mechanical $s$ - $d$ exchange interaction, causing giant Zeeman splitting [13] which is tunable in magnitude, sign and field dependence [14]. The constant $\alpha N_{0} \gg \Delta$ specifies the $s$ $d$ exchange strength and is the largest energy scale in the system. It hence has remained unclear whether FQHE states survive in the presence of magnetic impurities. Below we demonstrate that (i), the FQHE indeed exists in magnetic 2DESs, and (ii), that the opening and closing of gaps in an in-plane field can be described within a modified composite fermion (CF) picture, in which the $s$ - $d$ exchange is taken into account.

Let us first recall the CF-model which maps the FQHE onto the integer quantum Hall effect (IQHE) by introducing new particles, composite fermions, each composed of an electron and an even number (here: 2) of flux quanta [15]. Between $1<\nu<2$ the effective magnetic field for CFs vanishes at $\nu=3 / 2$ while they encounter an effective magnetic field $B_{C F}$ away from this filling [16]. In the vicinity of $\nu=3 / 2$ the CF filling factor $\nu^{\star}$ for composite fermions of holes is related to the one of electrons via $\nu=2-\nu^{\star} /\left(2 \nu^{\star} \pm 1\right)$ where ' \pm ' relates to $\mathrm{CF}$ filling factors at positive and negative effective field $B_{C F}$ [16]. Within this picture the fractions at $\nu=5 / 3$ and $4 / 3$ correspond to a filling of CF-LLs of $\nu^{\star}=1$ and 2 , 


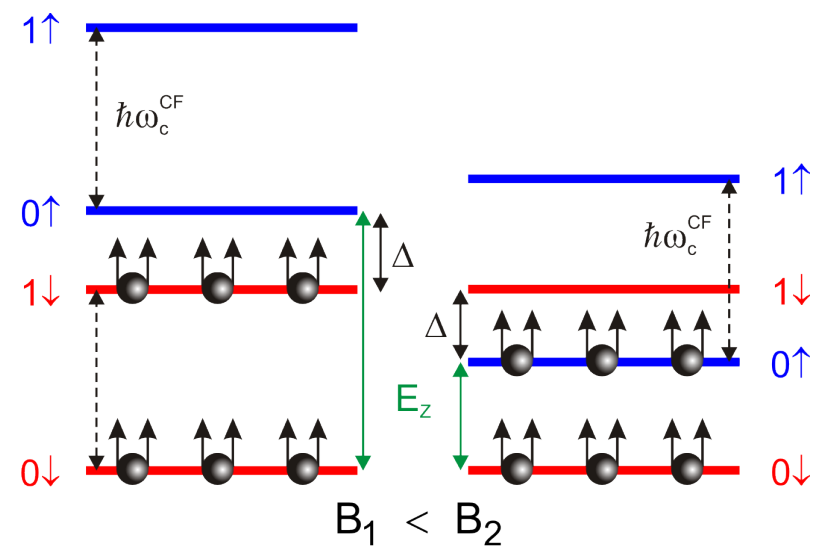

FIG. 1: CF-Landau levels at filling factor $\nu=4 / 3$. Within the CF picture, two (spin-split) CF-LLs are occupied $\left(\nu^{\star}=2\right)$. The gap $\Delta$ is the energy difference between highest occupied and lowest unoccupied CF-LL. The CF cyclotron energy $\hbar \omega_{c}^{C F}$, gap $\Delta$, and the Zeeman splitting $E_{Z}$ are indicated. With growing in-plane $B$ (right panel) $E_{Z}$ decreases, causing a change of the ground state's spin polarization, different to what is expected for non-magnetic materials.

respectively. Oscillations of $\rho_{x x}$ and steps in $\rho_{x y}$ around $\nu=3 / 2$ then reflect Shubnikov-de Haas (SdH) oscillations and the IQHE of CFs which occupy CF-LLs separated by energy gaps $\Delta=\hbar \omega_{c}^{C F}$ with $\omega_{c}^{C F}=e B_{C F} / m_{C F}$ ( $m_{C F}=$ composite fermion mass). Fig. 1 illustrates CF-LLs in $\mathrm{Cd}_{1-x} \mathrm{Mn}_{x}$ Te at fixed $B_{\perp}$ for two values of the in-plane magnetic field.

Our samples consist of $30 \mathrm{~nm}$ wide diluted magnetic $\mathrm{Cd}_{1-x} \mathrm{Mn}_{x}$ Te quantum wells sandwiched between $\mathrm{Cd}_{0.71} \mathrm{Mg}_{0.29} \mathrm{Te}$ barriers; the wells are single-sided modulation doped with iodine. Several $\mathrm{Cd}_{1-x} \mathrm{Mn}_{x}$ Te quantum structures from two different wafers (Mn concentrations of $x=0.24 \%$ and $0.30 \%$ ) have been studied. Here, the Mn concentration is obtained from the beating pattern of SdH oscillations at low $B$ [17] (Fig. 2b). Below we focus on data obtained from one sample with especially well developed FQHE states, having $x=0.24 \%$, a low-temperature mobility and carrier density of $\mu=115000 \mathrm{~cm}^{2} / \mathrm{Vs}$ and $n_{s}=3.95 \cdot 10^{11} \mathrm{~cm}^{-2}$, respectively, after illumination with a yellow LED. Illumination turned out to be crucial to observe fractional states as it increases the quantum scattering time $\tau_{q}$ by a factor of 5 to a value of $\sim 3$ ps. It is thus comparable to the values observed in GaAs and Si based 2DESs (see Fig. 2c) [18]. Measurements were done on rectangular samples of size $1.5 \times 3 \mathrm{~mm}^{2}$ with alloyed indium contacts in a ${ }^{3} \mathrm{He} /{ }^{4} \mathrm{He}$ dilution refrigerator with in-situ sample rotation stage.

Fig. 2a depicts $\rho_{x y}$ and $\rho_{x x}$ of our $\mathrm{Cd}_{1-x} \mathrm{Mn}_{x}$ Te quantum well (QW) device in perpen- 
dicular magnetic field for different temperatures T. Pronounced minima corresponding to $\nu=5 / 3,8 / 5$ and $4 / 3$ emerge in the lowest LL at low $T$ around $\nu=3 / 2$. No minimum is observed at $\nu=7 / 5$. A set of $\rho_{x x}$ data, taken at $T=25 \mathrm{mK}$ for different tilt angles, is shown in Fig. 2d. Tilting the sample by an angle $\theta$ yields the perpendicular magnetic field as $B_{\perp}=B \cdot \cos \theta$. A rather complex angular dependence appears in the $\rho_{x x}(\theta)$ traces, i.e. minima disappear and reappear. The $\nu=5 / 3$ minimum for instance starts - in clear contrast to non-magnetic 2DESs - to weaken continuously as soon as the sample is tilted away from the initial $\theta=0^{\circ}$ position, completely vanishes at about $\theta=30^{\circ}$ and reemerges upon further tilt. This is in stark contrast to the angular dependence of the $5 / 3$ minimum in GaAs or CdTe where no weakening of the gap was found. Notably, the $\nu=7 / 5$ minimum, absent in perpendicular magnetic field, appears around $\theta=38^{\circ}$.

To quantify our observations we performed angular dependent activation energy measurements, shown in Fig. 2e for the $\nu=5 / 3$ state. There, $\rho_{x x}$ at $\nu=5 / 3$ is recorded as a function of temperature for various $\theta$ (i.e. for the same $B_{\perp}$ ). Activation gaps $\Delta_{5 / 3}$ are then obtained from Arrhenius plots. Corresponding data of $\Delta_{5 / 3}$ are shown in Fig. 3a (top panel) as function of the total field $B$ : The gap $\Delta_{5 / 3}$ starts to close when increasing $B$ by tilting the sample, vanishes at around $11.45 \mathrm{~T}\left(\theta \approx 30^{\circ}\right)$, opens again and reaches a maximum value at $14.1 \mathrm{~T}\left(\theta \approx 45^{\circ}\right)$. The magnetic field at which the gap vanishes is obtained by extrapolating the data points left and right of the minimum. This gives $B=11.45 \mathrm{~T}\left(\theta \approx 30^{\circ}\right)$ [19]. This behavior, i.e. closing and opening of the gap is in line with the observed disappearance and reappearance of the $\nu=5 / 3$ minimum presented in Fig. 2 d.

To model the $\Delta_{\nu}(B)$ characteristics we expand the CF model [15] to cover diluted magnetic semiconductors (DMSs). To account for fractional states in a DMS we put forward a CF-LL fan chart modified by $s-d$ exchange interaction,

$$
E_{N, \uparrow \downarrow}=\left(N+\frac{1}{2}\right) \hbar \omega_{c}^{C F} \pm \frac{1}{2}\left[g^{\star} \mu_{B} B+\alpha N_{0} x S \mathcal{B}_{S}\right]
$$

The first term represents the cyclotron energy of a CF in the $N^{\text {th }}$ CF-LL, the second one describes Zeeman splitting in the presence of magnetic impurities [13]. It has two contributions: the first part, linear in $B$, is the conventional Zeeman term with $g^{\star}$, viewed as the $g$-factor of CFs. The second part is due to exchange between CFs and manganese spins with $S=5 / 2$, described by the $s$ - $d$ exchange constant $\alpha N_{0}$ and the Brillouin function $\mathcal{B}_{S}$. Since $\mathcal{B}_{S}$ saturates at $B$-fields around $1 \mathrm{~T}$ at low $T$, the exchange contribution to spin splitting is 

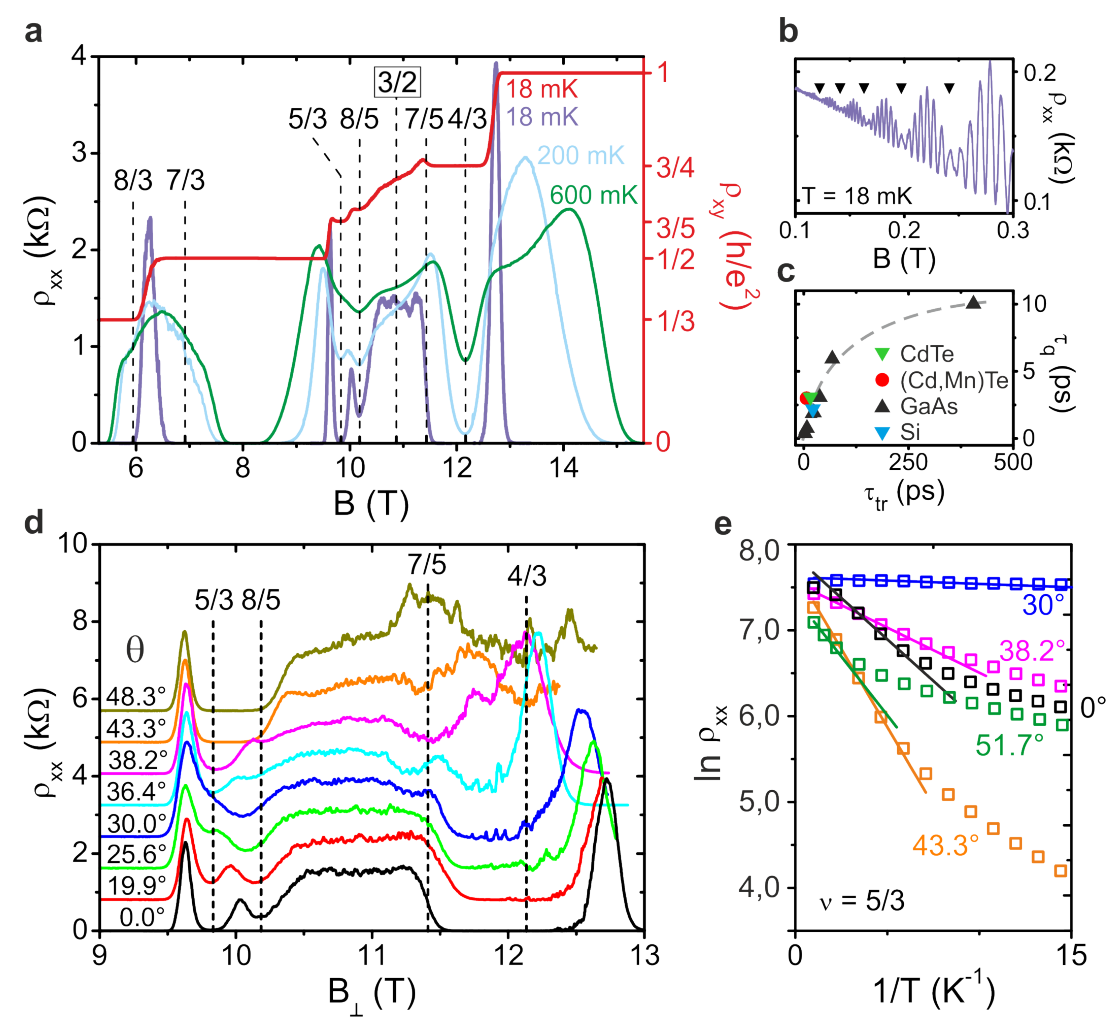

FIG. 2: (a) $\rho_{x y}$ and $\rho_{x x}$ at various temperatures after illumination. The $B$-field is applied perpendicular to the 2DES, filling factors are indicated. (b) Low field $\rho_{x x}$ data at $T=18 \mathrm{mK}$. The distinct beating pattern stems from the relative shift of spin-up and spin-down LLs due to the giant Zeeman splitting and is used to determine the Mn-concentration $x$. (c) Quantum scattering time $\tau_{q}$, extracted from the low field damping of $\mathrm{SdH}$ oscillations vs. momentum relaxation time $\tau_{t r}$ for different systems. Data for Si taken from [28], for GaAs from [29, 30] and for CdTe from [11]. $\tau_{t r}$ and $\tau_{q}$ of our $(\mathrm{Cd}, \mathrm{Mn}) \mathrm{Te} \mathrm{QW}$ fit nicely in the evolution of these parameters. The dashed line is a guide to the eye. (d) Angular dependence of $\rho_{x x}$ as function $B_{\perp}$ in the vicinity of $\nu=3 / 2$ at $T=25 \mathrm{mK}$. With increasing tilt angle $\theta$ the in-plane component of the magnetic field $B_{\|}=B \sin \theta$ increases, thus changing $E_{Z}$. Here, $B$ is the total applied field strength. Traces are shifted for clarity. (e) $\rho_{x x}$ vs. $1 / T$ on a semi-logarithmic scale for $\nu=5 / 3$ at various tilt angles $\theta$. Activation energies are extracted from Arrhenius plots (solid lines).

constant for fields above $10 \mathrm{~T}$, applied here. As the $g$-factor of electrons in CdTe is negative while the exchange contribution is positive $[13,20], E_{Z}$ decreases above $1 \mathrm{~T}$ and eventually vanishes if both terms are of equal strength, causing an unusual ordering of CF-LLs. The transition from a polarized fractional ground state to an unpolarized one with increasing 
in-plane field (sketched in Fig. 1) is one example of such an unusual ordering.

To retrace the $\Delta_{\nu}(B)$ characteristics we first adjust the CF-LL schemes to the experimental data at $5 / 3$ filling. As illustration, we show in the bottom panel of Fig. 3a the CF-LL scheme of the $\nu=5 / 3\left(\nu^{\star}=1\right)$ state as a function of $B$. The gap $\Delta_{5 / 3}$ (gray shaded region) is given by the energy difference between the CF-LLs above and below the Fermi energy $E_{F}^{C F}$. The bottom line here is that a vanishing gap at a specific $B$-value coincides with the crossing of spin-up and -down, $\mathrm{N}=0$, CF-LLs while a maximum gap corresponds to the separation between $N=0$ and $N=1$ CF-LLs with the same spin. By properly assigning $g^{\star}, \hbar \omega_{c}^{C F}$ and $\alpha N_{0}$, the magnetic field positions (for fixed $B_{\perp}$ ) at which a gap $\Delta_{\nu}$ opens or closes can be described quantitatively correct.

To compare model and experiment we assume that $\alpha N_{0}=220 \mathrm{meV}$ is the same for electrons and CFs. In case of $\nu=5 / 3$ the vanishing gap can be ascribed to the crossing of the $0, \uparrow$ and $0, \downarrow$ CF-LLs, occurring at vanishing spin splitting, so that $E_{Z}=g^{\star} \mu_{B} B+\alpha N_{0} x S=0$. Using that and the value at which the gap vanishes, $B=11.45 \mathrm{~T}, x=0.24 \%$ and $S=5 / 2$ we obtain $g^{\star}=-1.99$. This value deviates by about $16 \%$ from the $g$-factor of electrons in CdTe, $g=-1.67$; similar matching between $g$-factors of electrons and CFs has been seen in experiments on GaAs heterostructures [16]. Having fixed $g^{\star}=-1.99$ and $\alpha N_{0}=220 \mathrm{meV}$, we now use the "coincidence method" to determine $\hbar \omega_{c}^{C F}$. The coincidence of the $0, \uparrow$ and $1, \downarrow$ level occurs when the gap $\Delta_{5 / 3}$ reaches its maximal value at $B=14.1 \mathrm{~T}$ (see Fig. 3a). Then, we have that $E_{Z}(B=14.1 \mathrm{~T})=\hbar \omega_{\mathrm{c}}^{C F}$ and obtain $\hbar \omega_{c}^{C F}=3.54 \mathrm{~K}\left(m_{C F}=1.25 m_{e}\right)$ at $\nu=5 / 3$. The calculated evolution of $\Delta_{5 / 3}(B)$ (middle panel of Fig. 3a) reproduces the experimental data well for tilt angles below $\theta \sim 45^{\circ}$. With increasing in-plane field (tilt angle), however, the model describes the data less perfect. In Fig. 3a (middle panel) the model predicts a constant gap above $14 \mathrm{~T}$ while the data deviate. This is to some extent due to the larger error in extracting the gap; however, a reduction of the gap is also expected from the coupling of the growing in-plane field to the orbital motion of the electrons in our $30 \mathrm{~nm}$ wide QW [21]. The gap $\hbar \omega_{c}^{C F}$, obtained by the "coincidence method" is for $\nu=5 / 3$ (and also for $\nu=4 / 3$, see below) by a factor of $\sim 3$ larger than the activation gaps. Generally, gaps are overestimated by theory when disorder, LL mixing and finite thickness correction are neglected [22-25]. The difference in gap size reflects the different experimental techniques used to extract $\hbar \omega_{c}^{C F}$ : The fitting of the CF-LL spectrum (red dashed lines in Fig. 3a,b), obtained from the coincidence of different CF-LLs, is less affected by disorder. 


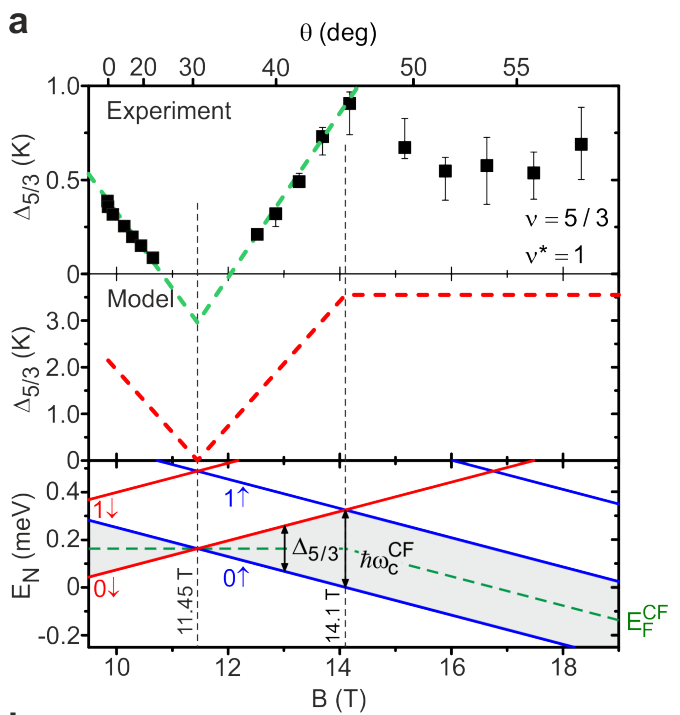

b

$\theta$ (deg)
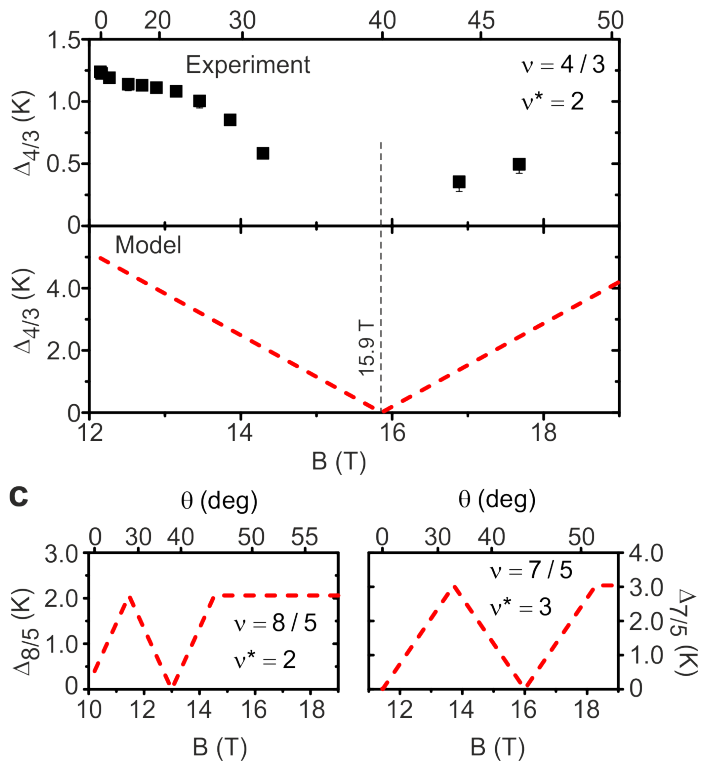

FIG. 3: (a) (Top panel) Activation gaps $\Delta_{5 / 3}(B)$, taken at fixed $B_{\perp}=9.85 \mathrm{~T}$ as function of total field $B$ (tilt angle $\theta$ ). The $B$-field position at which $\Delta_{5 / 3}$ closes was obtained by extrapolation (green dashed line). (Middle panel) Modeled gap $\Delta_{5 / 3}(B)$ as deduced from the CF-LL scheme of the $\nu=5 / 3\left(\nu^{\star}=1\right)$ state shown in the bottom panel. (Bottom panel) CF-LLs (Eq. 1) as function of total field $B$ for fixed $\hbar \omega_{c}^{C F}$, i.e. fixed $B_{\perp}$. The vanishing gap at $B=11.45 \mathrm{~T}$ is assigned to $N=0_{\downarrow}$ and $0_{\uparrow}$ CF-LL crossings while the maximum $\Delta_{5 / 3}$ at $B=14.1 \mathrm{~T}$ corresponds to the cyclotron gap $\hbar \omega_{c}^{C F}$. (b) Activation gap $\Delta_{4 / 3}$ (top) and corresponding model $\Delta_{4 / 3}$ (bottom). (c) Calculated evolution of the gaps $\Delta_{8 / 5}(B)$ and $\Delta_{7 / 5}(B)$ for the parameters given in the text. 
The cyclotron gap extracted from activated transport (top panels), in contrast, is strongly affected by disorder broadening and hence smaller [26].

Assuming that the usual CF-LL spectrum gets modified by the exchange energy $\alpha N_{0}=$ $220 \mathrm{meV}$ enables us to model the gap evolution $\Delta_{5 / 3}(B)$ quite reasonably. Below we check whether the modified CF-LL spectrum, extracted above is consistent with the observations made at other filling. For that we assume that $\alpha N_{0}=220 \mathrm{meV}$ and $g^{\star}=-1.99$ are independent of $\nu$. The latter assumption is justified by previous work on GaAs where the CF g-factor was found to be independent of $\nu$ and close to the one of electrons [16, 27]. In other words, we assume that the Zeeman gap closes at the same $B=11.45 \mathrm{~T}$ for all $\nu$. For $\nu=4 / 3$ we extract activation gaps of order $1 \mathrm{~K}$ for small $\theta$ (top panel, Fig. $3 \mathrm{~b}$ ) which decrease for higher tilt angles. For $\theta$ between $35^{\circ}$ and $42^{\circ}$ maxima emerge at $\nu=4 / 3$ so that we refrain from extracting activation energies in the region where the gap closes. At still higher tilt angles the gap reappears and the data allow to extract some activation gaps although the traces in Fig. 2d display some noise. The overall dependence of $\Delta_{4 / 3}(B)$, modeled with the same parameters $\alpha N_{0}$ and $g^{\star}$ as above (Fig. 3b, bottom panel) agrees well with the data plotted in the top panel. This time, however, we assume that $\hbar \omega_{c}^{C F}(\nu=4 / 3)=6 \mathrm{~K}$ $\left(m_{C F}=0.94 m_{e}\right)$. Here, the $B$-field at which $\Delta_{4 / 3}$ vanishes defines the CF cyclotron gap. We note, though, that the data points do not allow to determine the $B$-value at which the gap closes accurately. We have chosen $B=15.9 \mathrm{~T}$; shifting this point by a Tesla towards smaller fields reduces $\hbar \omega_{c}^{C F}$ by about $20 \%$ and leads a corresponding rescaling of the energy axis. Summarizing this point we note that the evolution of $\Delta_{4 / 3}(B)$ is consistent withthe CF-LL scheme deduced above.

Finally, let us now turn to the gaps at $\nu=7 / 5$ and $8 / 5$. While we observed gaps for $\nu=5 / 3$ and $4 / 3$ in different samples, fractional states at $7 / 5$ and $8 / 5$ filling were only observed in the sample discussed here. Because the extraction of gaps might be disputable since for most tilt angles no clear $\rho_{x x}$ minima arise we only show modeled $\Delta_{7 / 5}(B)$ and $\Delta_{8 / 5}(B)$ traces to illustrate that their evolution is, within experimental accuracy, consistent with the resistivity as function of $\theta$. Here we assume that maxima in the resistivity, measured as a function of $\theta$ (i.e. the resistivity along the dashed lines in Fig. 2d) correspond to vanishing CF-LL gaps. Using, as before, $\alpha N_{0}=220 \mathrm{meV}$ and $g^{\star}=-1.99$ we can obtain a vanishing gap at $\sim 13 \mathrm{~T}$ for filling factor $8 / 5$ in Fig. $3 \mathrm{c}$ (left panel). This agrees with a maximum of $\rho_{x x}$ at $\theta \sim 38^{\circ}$ in Fig. 2d. For $\nu=7 / 5$ maxima in $\rho_{x x}$ at $\theta \sim 8^{\circ}$ (not shown) 
and $\sim 48^{\circ}$ correspond to vanishing gaps at about $\sim 11.5 \mathrm{~T}$ and $17 \mathrm{~T}$ in Fig. 3c (left panel). This agrees reasonably well with the calculated traces.

In summary we note that without taking $\alpha N_{0}$ in Eq. (1) into account the angular dependence of $\Delta_{\nu}(B)$ cannot be reproduced. Especially the closing and opening of the $\nu=5 / 3$ gap, not observed in any other material, and corresponding to a change in spin polarization from $0, \downarrow$ to $0, \uparrow$ at $B=11.45 \mathrm{~T}$, highlights the impact of exchange splitting on the spin-polarization of the $\mathrm{CF}$ ground states.

We thank A. Wójs for fruitful discussions. This work was supported by the German Science Foundation (DFG) via Grants GI539, WE2476 and by the European Commission within the programme "Translational Access" (Contract 228043-EuroMagNET II). The research in Poland was partially supported by the National Science Centre (Poland) under grant DEC-2012/06/A/ST3/00247. 
[1] D. C. Tsui, H. L. Stormer, and A. C. Gossard, Phys. Rev. Lett. 48, 1559 (1982).

[2] R. B. Laughlin, Phys. Rev. Lett. 50, 1395 (1983).

[3] J. S. Xia, W. Pan, C. L. Vicente, E. D. Adams, N. S. Sullivan, H. L. Stormer, D. C. Tsui, L. N. Pfeiffer, K. W. Baldwin, and K. W. West, Phys. Rev. Lett. 93, 176809 (2004).

[4] R. G. Clark, S. R. Haynes, A. M. Suckling, J. R. Mallett, P. A. Wright, J. J. Harris, and C. T. Foxon, Phys. Rev. Lett. 62, 1536 (1989).

[5] J. P. Eisenstein, H. L. Stormer, L. Pfeiffer, and K. W. West, Phys. Rev. Lett. 62, 1540 (1989).

[6] R. Willett, J. P. Eisenstein, H. L. Störmer, D. C. Tsui, A. C. Gossard, and J. H. English, Phys. Rev. Lett. 59, 1776 (1987).

[7] G. S. Boebinger, H. L. Stormer, D. C. Tsui, A. M. Chang, J. C. M. Hwang, A. Y. Cho, C. W. Tu, and G. Weimann, Phys. Rev. B 36, 7919 (1987).

[8] K. I. Bolotin, F. Ghahari, M. D. Shulman, H. L. Stormer, and P. Kim, Nature 462, 196 (2009).

[9] A. Tsukazaki, S. Akasaka, K. Nakahara, Y. Ohno, H. Ohno, D. Maryenko, A. Ohtomo, and M. Kawasaki, Nature Mater. 9, 889 (2010).

[10] K. Lai, W. Pan, D. C. Tsui, S. Lyon, M. Mühlberger, and F. Schäffler, Phys. Rev. Lett. 93, $156805(2004)$.

[11] B. A. Piot, J. Kunc, M. Potemski, D. K. Maude, C. Betthausen, A. Vogl, D. Weiss, G. Karczewski, and T. Wojtowicz, Phys. Rev. B 82, 081307 (2010).

[12] R. N. McFarland, T. M. Kott, L. Sun, K. Eng, and B. E. Kane, Phys. Rev. B 80, 161310 (2009).

[13] J. K. Furdyna, Journal of Applied Physics 64, R29 (1988).

[14] F. J. Teran, M. Potemski, D. K. Maude, T. Andrearczyk, J. Jaroszyński, T. Wojtowicz, and G. Karczewski, Phys. Rev. B 82, 245120 (2010).

[15] J. K. Jain, Phys. Rev. Lett. 63, 199 (1989).

[16] R. R. Du, A. S. Yeh, H. L. Stormer, D. C. Tsui, L. N. Pfeiffer, and K. W. West, Phys. Rev. Lett. 75, 3926 (1995).

[17] F. J. Teran, M. Potemski, D. K. Maude, T. Andrearczyk, J. Jaroszynski, and G. Karczewski, Phys. Rev. Lett. 88, 186803 (2002). 
[18] We note that this scattering time is similar to the one measured in CdTe [11], demonstrating that incorporation of $\mathrm{Mn}$ does not, at least for small enough $x$, degrade the quality of the samples significantly.

[19] The negative gap at the crossing point can be ascribed to the finite CF Landau level width.

[20] S. D. Ganichev, S. A. Tarasenko, V. V. Bel'kov, P. Olbrich, W. Eder, D. R. Yakovlev, V. Kolkovsky, W. Zaleszczyk, G. Karczewski, T. Wojtowicz, and D. Weiss, Phys. Rev. Lett. 102, $156602(2009)$.

[21] B. A. Piot, D. K. Maude, U. Gennser, A. Cavanna, and D. Mailly, Phys. Rev. B 80, 115337 (2009).

[22] C. R. Dean, B. A. Piot, P. Hayden, S. Das Sarma, G. Gervais, L. N. Pfeiffer, and K. W. West, Phys. Rev. Lett. 100, 146803 (2008).

[23] A. Wójs and J. J. Quinn, Phys. Rev. B 74, 235319 (2006).

[24] F. C. Zhang and S. Das Sarma, Phys. Rev. B 33, 2903 (1986).

[25] R. H. Morf, N. d'Ambrumenil, and S. Das Sarma, Phys. Rev. B 66, 075408 (2002).

[26] A similar discrepancy has been observed for gaps between spin-split LLs in GaAs heterojunctions. D. R. Leadley, R. J. Nicholas, J. J. Harris, and C. T. Foxon, Phys. Rev. B 58, 13036 (1998).

[27] R. R. Du, A. S. Yeh, H. L. Stormer, D. C. Tsui, L. N. Pfeiffer, and K. W. West, Phys. Rev. B 55, R7351 (1997).

[28] K. Lai, W. Pan, D. C. Tsui, S. A. Lyon, M. Mühlberger, and F. Schäffler, Phys. Rev. B 72, $081313(2005)$.

[29] P. T. Coleridge, Phys. Rev. B 44, 3793 (1991).

[30] D. Zumbühl, private communication. 